\title{
SEMICONTINUOUS GROUPS AND SEPARATION PROPERTIES
}

\author{
ELLEN CLAY, BRADD CLARK and VIC SCHNEIDER
}

Department of Mathematics

The University of Southwestern Louisiana

Lafayette, LA 70504-1010

(Received December 31, 1990)

In 1948, Samuel [2] pointed out that the intersection of two group topologies need not be a group topology. However, a number of properties that hold for a group topology still hold for a topological space that is an intersection of group topologies. In order to study these properties, we shall describe a class of topologies that can be placed on a group which we call semicontinuous topologies. (We point out here that Fuchs [1] calls these spaces semitopological groups).

One important attribute of topological groups is separation. In particular, a topological group is Hausdorff if and only if the identity is a closed subset. While this is not true for semicontinuous groups, we shall see that an interesting "echo" of this property is true.

For each group $G$ we have a bijection inv: $G \rightarrow G$ defined by inv $(x)=x^{-1}$. Also for any fixed $a \in G$ we have bijections $1_{a}: G \rightarrow G$ defined by $1_{a}(x)=a x$ and $r_{a}: G \rightarrow G$ defined by $r_{a}(x)=x a$.

DEFINITION. A semicontinuous group is a group $G$ and a topology $\tau$ on $G$ making inv, $1_{a}$ and $r_{a}$ continuous for $a \in G$.

Clearly a semicontinuous group is a homogeneous space. Thus a great deal can be determined by considering a basis for the topology at the identity. In a manner analogous to that found in the theory of topological groups, one can demonstrate the following:

PROPOSITION 1. If $(G, \tau)$ is a semicontinuous group and $\varphi$ is a neighborhood base at the identity, then $\varphi$ satisfies

(i) If $U, V \in \mathcal{Y}$, then there exists $W \in \mathcal{\varphi}$ such that $W \subset U \cap U$.

(ii) If $a \in U$ and $U \in \varphi$, then there exists $V \in \mathcal{\varphi}$ such that $V a \subset U$.

(iii) If $U \in \Psi$ then there exists $V \in \varphi$ such that $V^{-1} \subset U$.

(iv) If $U \in \mathcal{\varphi}$ and $x \in G$ then there exists $V \in \mathcal{\varphi}$ such that $x V_{x}^{-1} \subset U$.

Furthermore, if $\varphi$ is any collection of subsets of $G$, each containing the identity, and $\varphi$ satisfies (i)-(iv) above, then there exists a unique semicontinuous topology $\tau$ on $G$ for which $\varphi$ is a neighborhood base at the identity. 
Any collection of subsets $\varphi$ satisfying (i)-(iv) is called a semifundamental system. Let $V=(-1,1)-\{x \mid x=r+\sqrt{2}$ and $r \in Q\} \subseteq R$ and let $W$ be the collection of all translation sets $a+V$ such that $0 \in a+V$. Finally let $\varphi$ be the collection of all finite intersections of elements of $W$.

A moment's reflection shows that $\mathcal{Y}$ is a semifundamental system that generates a topology $\tau$ which is finer than the usual topology on $R$. The set $Q$ is closed in $(R, \tau)$. Yet the quotient topology generated on $R / Q$ by projection from $(R, \tau)$ is the finite complement topology. Therefore the separation properties for semicontinuous groups are clearly different from those found in topological groups.

Another interesting example of a semicontinuous topology can be described as follows; let $B_{n}$ be the open ball of radius $1 / n$ centered at the origin of the plane, and let $V_{n}=B_{n}-\left\{(x, y) \mid 0<\frac{1}{n} x \leq y \leq n x\right\}$. The collection of sets $\left\{V_{n}\right\}_{n=2}^{\infty}$ forms a semifundamental system for the group $\left(R^{2},+\right)$. The relative topology on $\left(Q^{2},+\right)$ is an example of a second countable metric space that cannot be a topological group since no square of an open set can be placed inside $V_{n}$.

Let $(G, t)$ be a semicontinuous group and $m: G \times G \rightarrow G$ the multiplication map. We let $q(t)$ denote the quotient topology on $G$ generated by $m$ when the product topology $t \times t$ is placed on $G \times G$. If $N$ is a normal subgroup of $G$ and $(G, t)$ is a semicontinuous group, we shall denote the quotient topology on $G / N$ generated by the natural map $\pi: G \rightarrow G / N$, by $\pi(t)$.

LEMMA 2. If $(G, t)$ is a semicontinuous group, then both $m$ and $\pi$ are open maps and both $G / N$ and $(G, q(t))$ are semicontinuous groups.

PROOF. Let $U \times V$ be a basic open set in $t \times t$. Then $m^{-1}(m(U \times V))=\bigcup_{g \in G}\left(U g \times g^{-1} V\right)$. Therefore $m$ is an open map. Likewise $\pi^{-1}(\pi(u))=U N$ which is open in $(G, t)^{g}$ whenever $U \in t$. Thus $\pi$ is an open map.

Since $1_{a} \times$ id: $(G \times G, t \times t) \rightarrow(G \times G, t \times t)$ is continuous and $q(t)$ is a quotient topology, $1_{a}:(G, q(t)) \rightarrow(G, q(t))$ is continuous. Similar arguments show that the maps $r_{a}:(G, q(t)) \rightarrow(G, q(t))$ and inv: $(G, q(t)) \rightarrow(G, q(t))$ are continuous. The proof that the quotient topology on $G / N$ is semicontinuous is done in the same fashion.

LEMMA 3. If $S \subset G$ then $\bar{S}=\bigcap_{V \in \varphi} V S$.

PROOF. $x \notin \bigcap_{V \in \varphi} V S$ iff there exists $W \in \mathcal{Y}$ with $x \notin W S$ iff $W^{-1} x \cap S=\phi$.

THEOREM $4 . G / N$ is Hausdorff iff $N=\bigcap_{V \in \varphi} V^{2} N$.

PROOF. We consider the following commutative diagram:

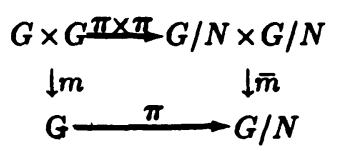

We have that $\left\{V^{2} \mid V \in \varphi\right\}$ is a semifundamental system for $q(t)$ whenever $\varphi$ is a semifundamental system for $t$. The identity element in $(G / N, \pi(q(t)))$ will be closed if and only if $N=\bigcap_{V \in \varphi} V^{2} N$. The identity element in $(G / N, q(\pi(t)))$ will be closed if and only if the diagonal is closed in $G / N \times G / N$. However $\pi(q(t))=q(\pi(t))$ since the maps are open.

COROLLARY 5. $(G, t)$ is Hausdorff if and only if $\bigcap_{V \in \mathcal{Y}} V^{2}=\{e\}$. 
COROLLARY 6. If $(G, t)$ is a minimal Hausdorff semicontinuous group then $(G, t)$ is topological group if and only if $\bigcap_{V \in \mathcal{Y}} V^{4}=\{e\}$.

We can define an equivalence relation on $(G, t)$ by defining $x \sim y$ if and only if there does not exist $V \in \varphi$ such that $x V \cap y V=\phi$. Let $K$ denote the equivalence class of $e$ under this equivalence relation. We call $K$ the Hausdorff Kernel of $(G, t)$. THEOREM 7. $K=\bigcap_{V \in \varphi} V^{2}$ and $K$ is the minimum normal subgroup with the property that
$G / K$ is Hausdorff.

PROOF. We note by Lemma 3 that $\bigcap_{V \in \varphi} V^{2}$ is the closure of $\{e\}$ in $(G, q(t))$. Therefore by an argument similar to that for topological groups, $\bigcap_{V \in \varphi} V^{2}$ is a normal subgroup of $G$. Since we can without loss of generality assume that $V$ is symmetric, it is clear the $K=\bigcap_{V \in \varphi} V^{2}$. The proof of Theorem 4 shows that $G / K$ is Hausdorff if and only if $K$ is closed in $(G, q(t))$. But $K$ is the smallest closed normal subgroup in $(G, q(t))$.

In a like manner we can define an equivalence relation on $(G, t)$ by declaring $x \sim y$ if and only if there does not exists a continuous function $\phi: G \rightarrow R$ with $\phi(x) \neq \phi(y)$. The equivalence class of $e$ under this relation will also be a closed normal subgroup that we call the completely Hausdorff kernel of $(G, t)$.

\section{REFERENCES}

1. FUCHS, L., Infinite Abelian Group, Vol. 1, Acad. Press, New York and London, 1970.

2. SAMUEL, P., "Ultrafilters and compactifications of uniform spaces," Trans. A.M.S. 64 (1948), 110-132. 


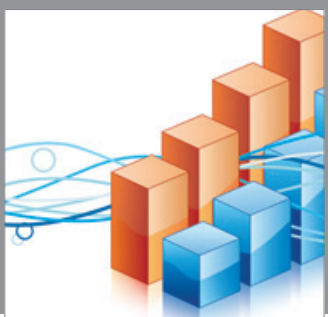

Advances in

Operations Research

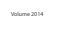

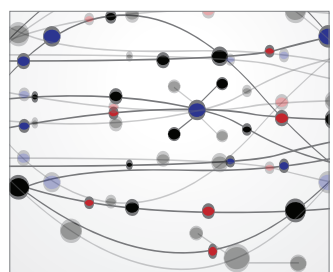

\section{The Scientific} World Journal
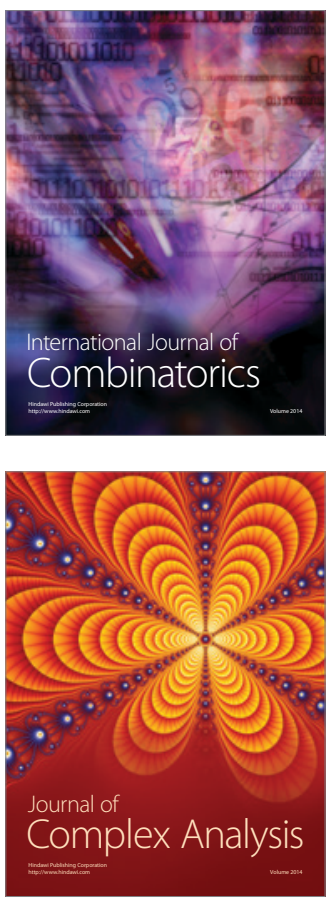

International Journal of

Mathematics and

Mathematical

Sciences
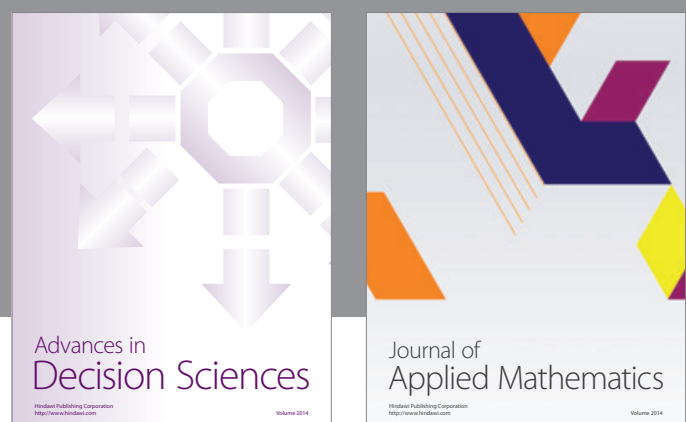

Journal of

Applied Mathematics
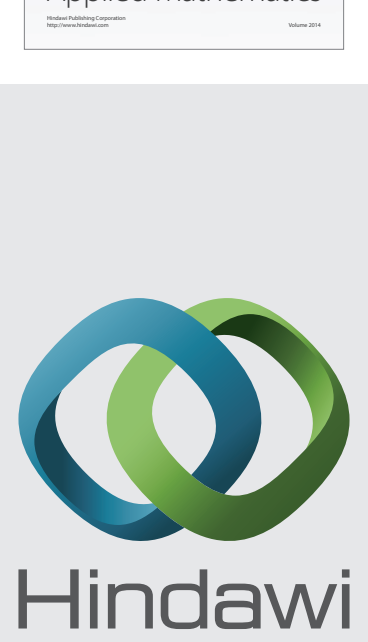

Submit your manuscripts at http://www.hindawi.com
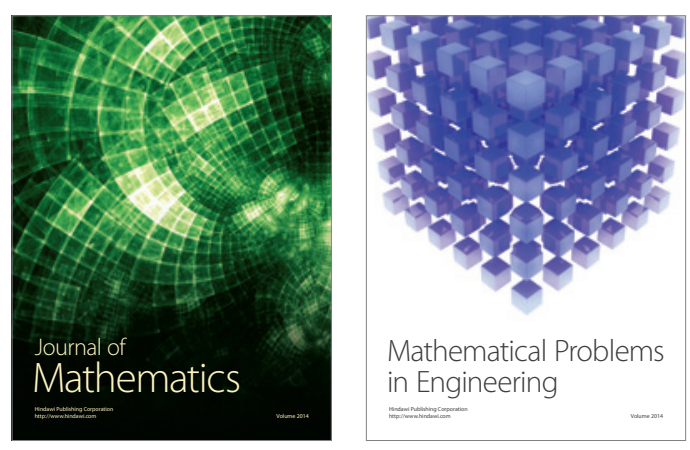

Mathematical Problems in Engineering
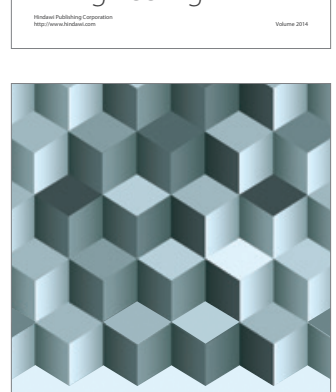

Journal of

Function Spaces
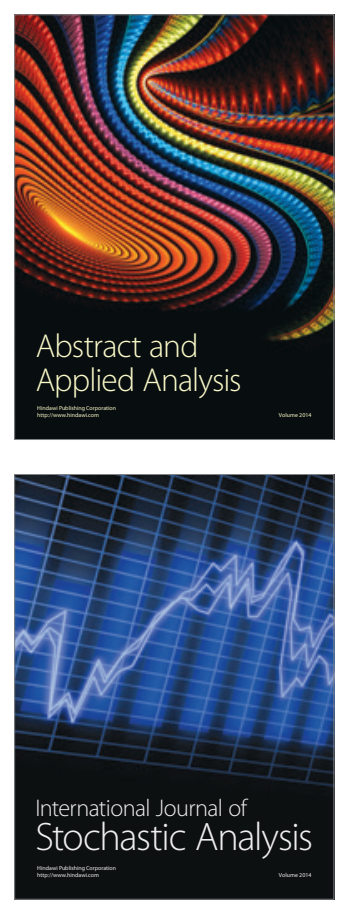

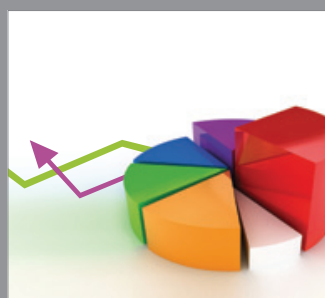

ournal of

Probability and Statistics

Promensencen
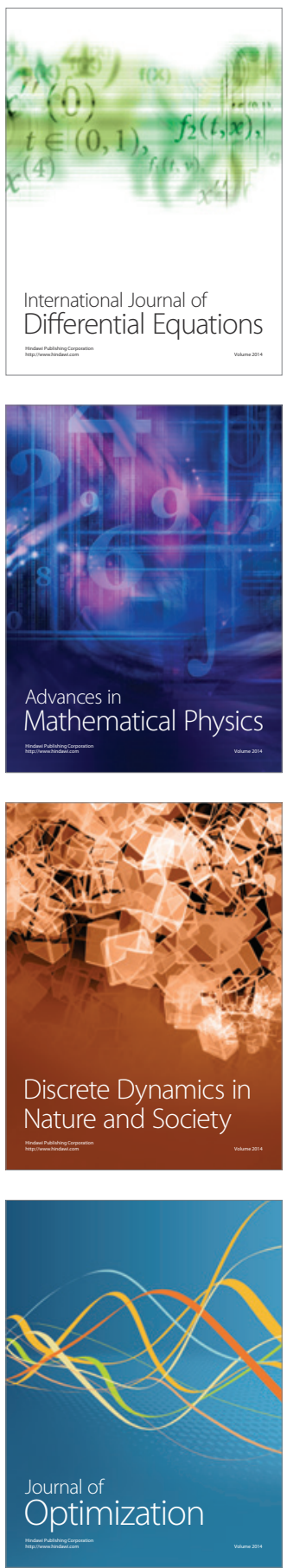